\title{
Transgene Stacking and Coordinated Expression of Plant Defensins Confer Fungal Resistance in Rice
}

\author{
Sanjay Jha $\cdot$ Bharat Bhushan Chattoo
}

Received: 23 May 2009 / Accepted: 14 August 2009 /Published online: 4 September 2009

(C) Springer Science + Business Media, LLC 2009

\begin{abstract}
Transgenic rice with cleavable chimeric polyprotein and single-protein gene constructs placed under the control of single maize ubiquitin promoter were generated by Agrobacterium tumefaciens-mediated transformation. The polyprotein precursor consists of a leader peptide and two different antimicrobial proteins (AMPs), Dm-AMP1 and Rs-AFP2, from the seeds of Dahlia merckii and Raphanus sativus, respectively. These genes were linked by a 16 amino acid Ib-AMP linker peptide region isolated from the seeds of Impatiens balsamina. Average expression of Dm-AMP1 and Rs-AFP2 was $8.35 \pm 0.56$ and $8.1 \pm 0.6$ $2 \mathrm{~g} / \mathrm{mg}$ of total soluble proteins in the leaf extracts of transgenic plants. Plants transformed with polyprotein construct showed significantly improved disease resistance against Magnaporthe oryzae and Rhizoctonia solani by $90 \%$ and $79 \%$, respectively, as compared to untransformed plants. The plants transformed with polyprotein construct were more resistant to $M$. oryzae compared to plants transformed with control single-gene constructs of DmAMP1 and Rs-AFP2. The chimeric polyprotein was cleaved and present in equimolar concentration in transgenic rice and the individual AMPs were secreted into the
\end{abstract}

S. Jha $\cdot$ B. B. Chattoo $(\bowtie)$

Centre for Genome Research,

Department of Microbiology and Biotechnology Centre,

Faculty of Science,

The M. S. University of Baroda,

Vadodara, Gujarat 390002, India

e-mail: bharat.chattoo@bcmsu.ac.in

Present Address:

S. Jha

Department of Biotechnology,

ASPEE College of Horticulture and Forestry,

Navsari Agricultural University,

Navsari, Gujarat 396450, India extracellular space. The results of in vitro, in planta, and microscopic analyses suggest that chimeric polyprotein expression has the potential to provide broad-spectrum disease resistance in rice.

Keywords Rs-AFP2 Dm-AMP1 - Magnaporthe oryzae . Rhizoctonia solani $\cdot$ Transgenic rice $\cdot$ Plant-microbe interactions · Polyprotein

\section{Introduction}

Rice is the staple food of over $50 \%$ of the world population. Rice blast (Magnaporthe oryzae) and sheath blight (Rhizoctonia solani) are among the most devastating fungal diseases that limit the productivity of rice $(\mathrm{Ou}$ 1985). Plants defend against antagonistic microorganisms using an array of antimicrobial compounds (Broekaert et al. 1995). Defensins are a class of evolutionarily and structurally related small, highly basic, cysteine-rich peptides, displaying a broad-spectrum antifungal activity in vitro (Osborn et al. 1995; Thomma et al. 2002). Since, defensins are the peptides with disulfide bridges; they have to pass through endoplasmic reticulum before secretion. It was shown that different plant defensins are able to interact with different sphingolipids. Dahlia plant defensin, Dm-AMP1 interacts with mannosylated sphingolipids, whereas the radish plant defensin Rs-AFP2 interacts with fungal glucosylceramides occurring in the outer plasma membrane (Thevissen et al. 2004), leading to increased $\mathrm{K}^{+}$efflux and $\mathrm{Ca}^{2+}$ influx and change in membrane potential (Osborn et al. 1995; Terras et al. 1992). Dm-AMP1 and Rs-AFP2 inhibited the growth of $M$. oryzae and $R$. solani in vitro, at micromolar concentration (Terras et al. 1995). Thus, co-expression of these 
antimicrobial proteins (AMPs) with different biochemical targets is an attractive approach for developing durable disease resistance against phytopathogens.

Conventionally, genetic engineering involves transformation of a crop plant with a single gene of interest (Ronald 1997; Zhang et al. 1998). However, in the context of disease resistance, since pathogens are able to overcome resistance to single genes in a short period, it is desirable to pyramid more than one gene to provide durable resistance (Mew et al. 1992). Availability of cloned disease resistance (Song et al. 1995) or defense genes (Huang et al. 1997; Velazhahan et al. 1998; Takakura et al. 2000; Osborn et al. 1995; Terras et al. 1995) and improved transformation tools and strategies (Hiei et al. 1994; Christou 1997; Chen et al. 1998; Kim et al. 2003) have made it possible to pyramid genes of disease resistance in crop plants. The capability of rice genome to integrate, co-express, and inherit several transgenes, without undergoing developmentally and reproductively unfavorable phenotypic changes has also been documented (Chen et al. 1998). The success of gene pyramiding is largely dependent upon the selection of the genotypes of the crop to be transformed and the genes to be employed. The combination of genes encoding proteins involved in pathogen recognition and/or subsequent activation of signaling pathways leading to defense responses (Van Loon and Van Strien 1999) and those exhibiting direct antimicrobial properties (Broglie et al. 1991; Yun et al. 1998) by virtue of their interaction with pathogen cell wall and cell membrane can be expected to generate plants with wide-spectrum resistance against pathogens.

Different strategies used to generate fungal disease resistant rice plants, include the use of antimicrobial genes or expression of plant disease signaling components (Lorito et al. 1998; Cao et al. 1998; Datta et al. 1999). The resistance obtained by a single gene is limited, as the degree of enhanced resistance is low, thus necessitating the use of various gene combinations (Jach et al. 1995). A number of approaches have been used to introduce transgenes in rice to get an adequate, durable, and broad-spectrum disease resistance (Ye et al. 2000; Datta et al. 2002; Maruthasalam et al. 2007). One of the major constraints in the coexpression of transgenes is their uncoordinated expression, even when the genes are physically linked (Maqbool and Christou 1999). Moreover, the presence of multiple copies of the same promoter within a transgenic plant often results in transcriptional silencing of the transgenes (Matzke and Matzke 1998). Present work provides an approach to overcome these problems by introducing the sequences of genes for different proteins in a single open reading frame via short linkers; subsequently, the linker sequences are cleaved into constituent protein units by host cell proteinase while passing through the plant endomembrane system (Francois et al. 2002).
A natural polyprotein Ib-AMP, present in the seeds of Impatiens balsamina (Tailor et al. 1997), consists of a leader peptide, followed by six 20 -amino-acid-long mature peptide domains, each flanked at either side by propeptide domains ranging from 16 to 35 amino acids in length. In the present study the fifth propeptide (16 amino acids) of the Ib-AMP polyprotein was used. We transformed Pusa basmati 1 (an elite indica rice variety, highly susceptible to both blast and sheath blight) with tripartite gene construct consisting of Dm-AMP1 with native signal peptide, 16amino-acid-linker peptide of the Ib-AMP precursor and RsAFP2 gene to evaluate their expression, processing, and ability to confer resistance against rice fungal pathogens.

\section{Results}

Characterization of transgenic rice lines

Plant transformation vector pUb1-DR-T was constructed for co-expression of Dm-AMP1 and Rs-AFP2 in rice (Fig. 1a). Dm-AMP1 (without termination codon) was translationally fused with the Ib-AMP1 linker peptide, which was further translationally fused with Rs-AFP2 coding region. In the expression product of pUb1-DR-T polyprotein construct, 16amino-acid Ib-AMP propeptide is flanked on either side by the mature protein domain of the Dm-AMP1 and Rs-AFP2 (50 and 51 amino acids, respectively). The expression product has a leader peptide ( 28 amino acids) with amino terminus derived from the Dm-AMP1 precursor. Single-gene constructs $\mathrm{pUb} 1-\mathrm{D}-\mathrm{T}$ and $\mathrm{pUb} 1-\mathrm{R}-\mathrm{T}$ were made with DmAMP1 leader peptide, in which the predicted expression product consisted of the Dm-AMP1 (Jha et al. 2009) and RsAFP2 mature proteins, respectively (Fig. 1a). Transgenic rice plants expressing cleavable chimeric polyprotein and singlegene constructs were generated via Agrobacterium-mediated transformation, using hygromycin phosphotransferase gene (hpt) as a plant selectable marker (Fig. 1a). A total of 150 scutellum-derived embryogenic calli were used as explants for pUb1-DR-T construct, out of which 80 (53.33\%) hygromycin-resistant calli were obtained. Among these, 27 $(33.75 \%)$ calli regenerated into whole plants and set seeds.

Twenty-seven pUbI-DR-T, 28 pUb1-D-T, and 28 pUb1R-T primary transformants $\left(\mathrm{T}_{0}\right)$ were obtained and analyzed. A 521-bp polymerase chain reaction (PCR)-amplified product corresponding to the gene sequence encoding the chimeric protein sequence was observed in all the 27 putative transformed plants and the positive control (pUbIDR-T plasmid), but was absent in the untransformed plants (Fig. 1b). Subsequently, mature leaves of $T_{2}$ transgenic lines were collected and checked for transgene integration by Southern blot analysis (Fig. 1c). Independent $T_{2}$ transgenic lines (pUbI-DR-T, pUbI-D-T, and pUbI-R-T) with single- 
a
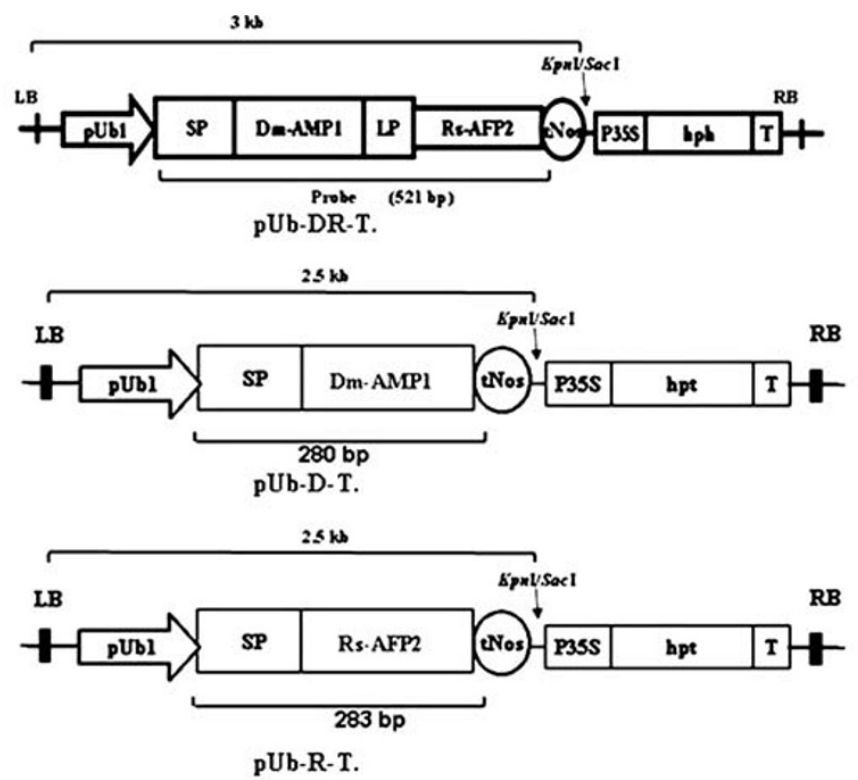

b
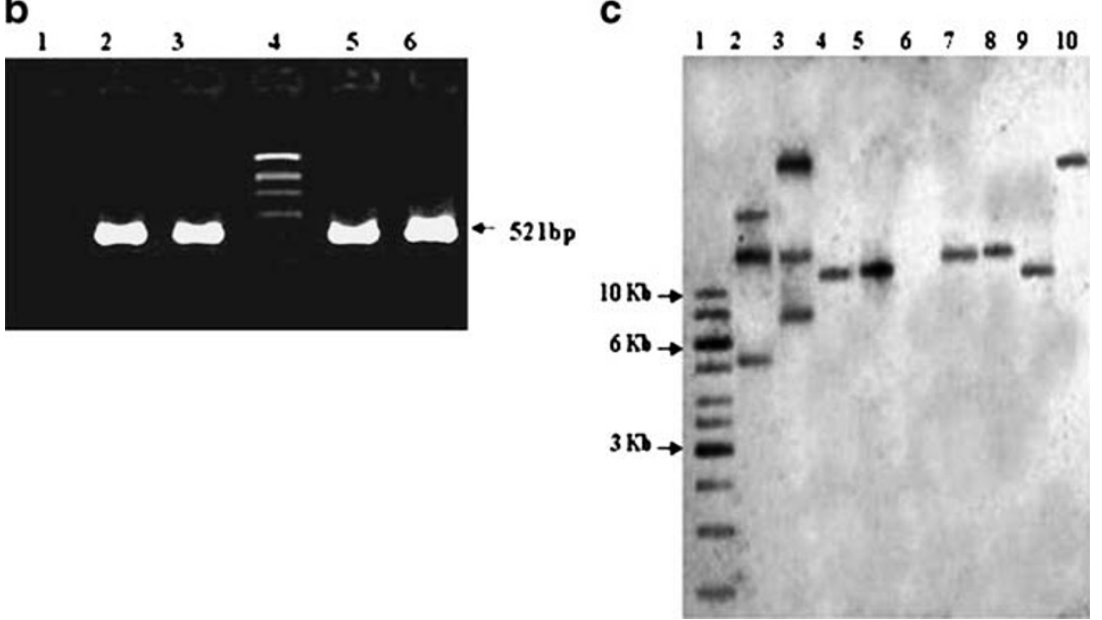

Fig. 1 a Plant expression vector pUb-DR-T, pUb-D-T, and pUb-R-T. pUb1 ubiquitin promoter, $S P$ signal peptide, tNos $3^{\prime}$ untranslated terminator region of the Agrobacterium tumefaciens nopaline synthase gene, $p 35 S$ CaMV35S promoter, hpt hygromycin phosphotransferase. b Total genomic DNA extracted from transgenic plants was amplified by PCR using specific primers for polyprotein (521-bp) coding region. Lane 1 negative control (untransformed Pusa basmati 1); lane 2 positive control (pUbI-DR-T plasmid); lane 3, 5, and 6 plants transformed with polyprotein gene construct; lane $41 \mathrm{~kb}$ ladder. c
Southern blot analysis of KpnI-digested genomic DNA from untransformed and representative polyprotein-transformed transgenic plants in $T_{2}$ generation. Genomic DNA $(10 \mu \mathrm{g})$ was digested with restriction enzymes and blotted onto nitrocellulose membranes after electrophoresis in a $0.8 \%$ agarose gel. The membrane was hybridized with 521bp polyprotein coding sequence; lane $11 \mathrm{~kb}$ Ladder; lane $2 \mathrm{DR}-\mathrm{T}-18$; lane 3 DR-T-2; lane 4 DR-T-14; lane 5 DR-T-11; lane 6 untransformed negative control; lane 7 DR-T-6; lane 8 DR-T-17; lane 9 DR$\mathrm{T}-4$; lane 10 DR-T-22. integration event were analyzed for expression at the mRNA level. Northern blot analysis using the coding region of chimeric gene as a probe indicated the presence of transcripts in leaves of lines transformed with pUbI-DR-T, pUbI-D-T, or pUbI-R-T, but not in untransformed lines (Fig. 2a). A clear difference in the size of mRNA in plants transformed with the polyprotein construct as compared to plants transformed with the single-protein gene construct could be observed. Quantification of band intensity in the lines transformed with pUbI-DR-T or with pUbI-D-T and pUbI-R-T indicated that transcripts levels did not differ significantly among different transgenic plants. The rice actin gene was taken as an internal control (Fig. 2a). Leaves of $\mathrm{T}_{2}$ plants were analyzed by Western blot to detect the presence of Dm-AMP1 and RsAFP2. Previously, we have successfully generated transgenic rice lines harboring gene for $R s-A F P 2$ (unpublished) and Dm-AMP1 (Jha et al. 2009). Dm-T-32 and Rs-T-42 lines showed highest expression of transgene product as well as improved resistance against $M$. oryzae and $R$. solani. Therefore, Dm-T-32 and Rs-T-42 lines were used as positive 


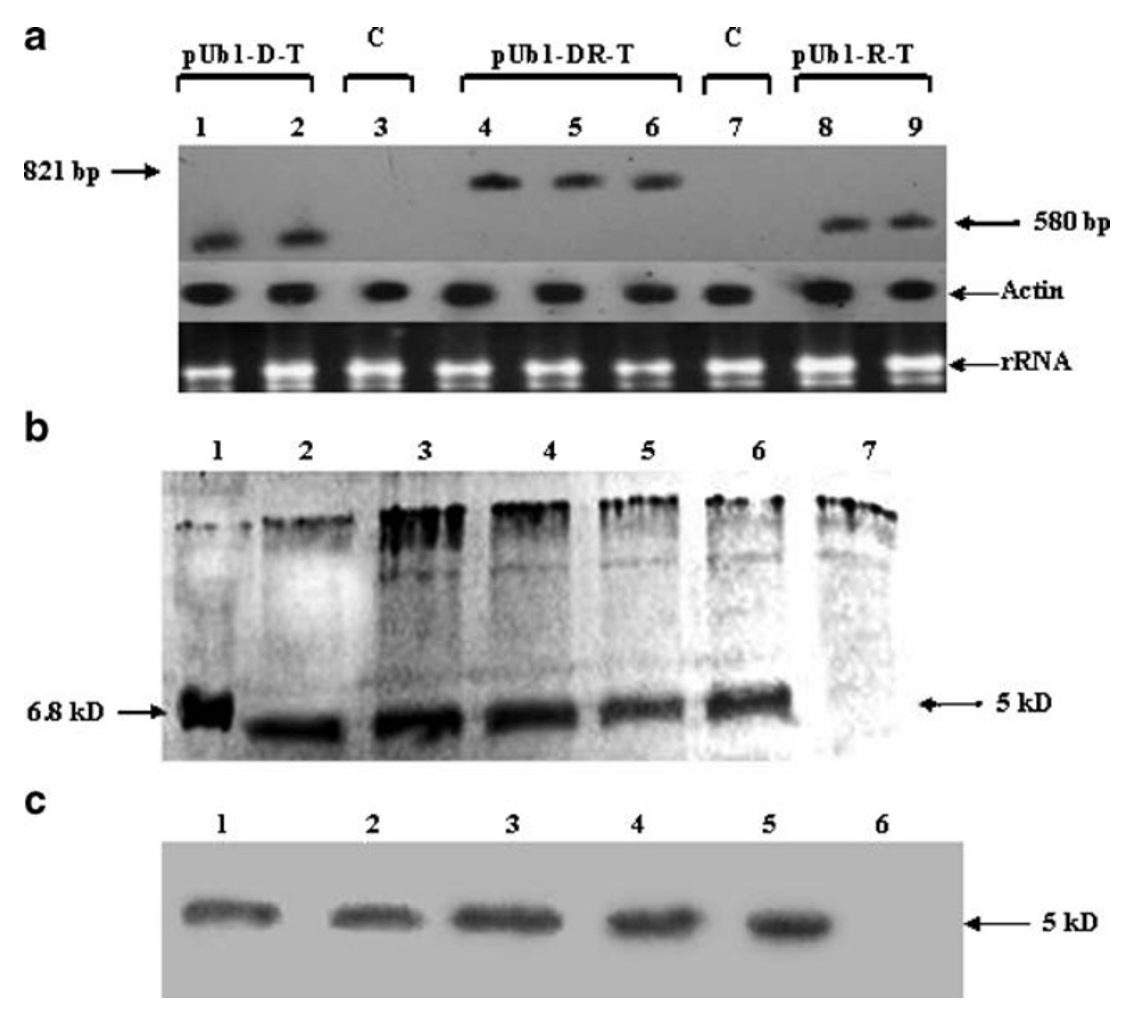

Fig. 2 a Northern blot analysis in leaves of transgenic $T_{2}$ generation lines transformed with constructs, pUb1-DR-T, pUb1-D-T, and pUb1$\mathrm{R}-\mathrm{T}$; lane $1 \mathrm{Dm}-\mathrm{T}-32$, lane $2 \mathrm{Dm}-\mathrm{T}-14$, lane 3 negative control (untransformed Pusa basmati 1), lane 4 DR-T-4, lane 5 DR-T-22, lane 6 DR-T-6, lane 7 negative control (untransformed Pusa basmati 1), lane $8 \mathrm{Rs}-\mathrm{T}-42$, lane 9 Rs-T-24. About $20 \mu \mathrm{g} /$ sample of total RNA were separated in a formaldehyde/agarose gel, transferred onto nylon membranes and hybridized to a non-radioactively labeled probe from the coding region of chimeric polyprotein gene (Dm-AMP1+RsAFP2). $C$ Negative control (untransformed rice plants); $p U b 1-D-T$ rice plants transformed with Dm-AMP1; $p U b 1-R-T$ Rice plants transformed with Rs-AFP2; $p U b 1-D R-T$ rice plants transformed with the

control in the experiment as and when required. Transformed plants showed the expression of Dm-AMP1 and/or Rs-AFP2 proteins co-migrating with their respective positive controls (Dm-T-32 and Rs-T-42), which was absent in the untransformed control (Fig. 2b, c). The amount of AMPs in leaves from the series of $\mathrm{T}_{2}$ rice plants transformed with pUbI-DR$\mathrm{T}$ construct was determined using enzyme-linked immunosorbent assay (ELISA). Average expression of Dm-AMP1 and Rs-AFP2 was 8.33 and $8.07 \mu \mathrm{g} / \mathrm{mg}$ of total soluble proteins in the leaf extracts of pUbI-DR-T transgenic lines (Table 1). Whereas, the average expression levels of the same transgene products were 4.96 and $4.93 \mu \mathrm{g} / \mathrm{mg}$ of total soluble proteins in pUbI-D-T and pUbI-R-T plants, respectively (Table 1). None of the transgenic lines showed any obvious phenotypic changes compared with control plants throughout the life cycle.

The expression of OsPR1a (gi: 9801265) gene has been widely used as an indicator for induction of defense responses against rice blast (Agrawal et al. 2001). To polyprotein construct. Actin was used as internal control. rRNA bands (28 and 18S) from an ethidium bromide-stained gel of the corresponding RNA samples indicate equivalent loading of RNA. b Immunodetection of Dm-AMP1 in transgenic rice lines transformed with polyprotein construct; lane 1 pre-stained marker (CPM1); lane 2 DR-T-22; lane 3 DR-T-6; lane 4 DR-T-4; lane 5 DR-T-11; lane 6 DmT-32 (positive control); lane 7 negative control (untransformed Pusa basmati 1). c Immunodetection of Rs-AFP2 in transgenic rice lines transformed with polyprotein construct; lane 1 DR-T-22; lane 2 DR-T6; lane 3 DR-T-4; lane 4 DR-T-11; lane 5 Rs-T-42 (positive control); lane 6 negative control (untransformed Pusa basmati 1).

explore a possible activation of the plant defense system in the transgenic rice lines transformed with pUbI-DR-T, pUbI-D-T, and pUbI-R-T construct, the expression of the endogenous PR-1a gene was analyzed at the RNA level, without challenging with the pathogen. The transcript for PR-1a was not detectable in transgenic lines (Fig. 3), but as expected, PR-1a transcripts were detected in M. oryzaeinfected control (Fig. 3).

Localization of the Dm-AMP1 and Rs-AFP2 protein in rice tissue

Subcellular localization of Dm-AMP1 and Rs-AFP2 in plants transformed with polyprotein gene construct was analyzed immunohistochemically. Two controls were run along with the test tissue samples from transgenic plants. One of the two control tissues was from an untransformed plant and was treated with both anti-Dm-AMP1/anti-RsAFP2 antibodies and Fluorescein isothiocyanate (FITC)- 
Table 1 Expression Levels and Disease Rating of Transgenic Lines

\begin{tabular}{|c|c|c|c|c|c|}
\hline \multirow[t]{2}{*}{ Constructs } & \multirow[t]{2}{*}{ Transgenic line } & \multicolumn{2}{|c|}{ Expression level ( $\mu \mathrm{g} / \mathrm{mg}$ of total protein) } & \multicolumn{2}{|l|}{ Disease rating } \\
\hline & & Dm-AMP1 & Rs-AFP2 & $\%$ DLA (M. oryzae) & $\%$ infection $(R$. solani $)$ \\
\hline \multicolumn{6}{|l|}{ pUbI-DR-T } \\
\hline & Untransformed & 0.00 & 0.00 & 69.68 & 66.33 \\
\hline & DR-T-11 & 7.40 & 7.20 & 14.38 & 25.00 \\
\hline & DR-T-6 & 8.15 & 8.03 & 13.12 & 20.83 \\
\hline & DR-T-4 & 8.60 & 8.40 & 13.00 & 14.58 \\
\hline & DR-T-22 & 9.15 & 8.70 & 9.89 & 14.38 \\
\hline & Average & 8.33 & 8.08 & 12.59 & 18.69 \\
\hline \multicolumn{6}{|l|}{ pUb1-D-T } \\
\hline & Untransformed & 0.00 & - & 69.68 & 66.33 \\
\hline & Dm-T-16 & 4.32 & - & 18.35 & 28.00 \\
\hline & Dm-T-21 & 5.04 & - & 10.74 & 18.67 \\
\hline & Dm-T-14 & 4.79 & - & 14.74 & 20.70 \\
\hline & Dm-T-32 & 5.69 & - & 10.12 & 18.58 \\
\hline & Average & 4.96 & - & 13.48 & 21.49 \\
\hline \multicolumn{6}{|l|}{ pUb1-R-T } \\
\hline & Untransformed & - & 0.00 & 69.68 & 66.33 \\
\hline & Rs-T-31 & - & 4.50 & 25.64 & 48.85 \\
\hline & Rs-T-26 & - & 4.80 & 15.17 & 43.08 \\
\hline & Rs-T-24 & - & 5.20 & 11.73 & 38.23 \\
\hline & Rs-T-42 & - & 5.29 & 11.01 & 36.38 \\
\hline & Average & - & 4.95 & 15.89 & 41.64 \\
\hline SE & & 0.18 & 0.10 & 0.66 & 1.11 \\
\hline $5 \% \mathrm{LSD}$ & & 0.53 & 0.19 & 1.87 & 3.17 \\
\hline
\end{tabular}

Levels of Dm-AMP1 and/or Rs-AFP2 in transgenic lines, Blast and sheath blight infection assay results (\% DLA and \% infection) were compared by one-way analysis of variance (ANOVA). Means were compared using LSD5\%. Design of experiment was completely randomized block design (CRD)

conjugated goat anti-rabbit IgG antibody. The other control tissue was from a transgenic plant and was exposed only to the FITC-conjugated goat anti-rabbit IgG antibody, in the absence of anti-Dm-AMP1/anti-Rs-AFP2 antibodies. Both, Dm-AMP1 and Rs-AFP2 were observed in the apoplast of leaves and roots of transformed rice, while cytoplasmic content remained unlabelled (Fig. 4 (i and ii)).

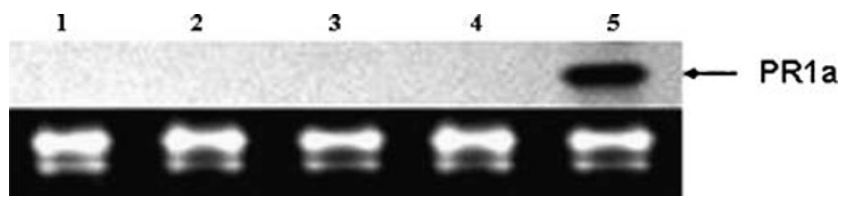

Fig. 3 Northern blot analysis of transgenic lines for the expression of rice OsPR-1a gene. About $20 \mu \mathrm{g} / \mathrm{sample}$ of total RNA were separated in a formaldehyde/agarose gel, transferred onto nylon membranes and hybridized to a non-radioactively labeled PR1a probe; lane 1 control, non-infected wild-type plants; lane 2 DR-T-6; lane 3 DR-T-4; lane 4 DR-T-11; lane 5 M. oryzae-infected untransformed Pusa basmati 1. b rRNA bands (28 and $18 \mathrm{~S}$ ) from an ethidium bromide-stained gel of the corresponding RNA samples indicate equivalent loading of RNA.
Disease tolerance of transgenic rice

Resistance to $M$. oryzae was evaluated on $\mathrm{T}_{2}$ transgenic lines (pUbI-DR-T, pUbI-D-T, pUbI-R-T) and the untransformed control. All the transgenic lines showed significant increase in resistance to blast infection. When transgenic plants were challenged with $M$. oryzae, remarkable reduction in diseased leaf area (\% DLA) was observed between untransformed (69.68\%), pUb1-DR-T (11.83\%), pUb1-D-T (13.49\%), and pUb1-R-T (15.72\%) transgenic lines at 5\% LSD (Fig. 5a and Table 1). Similarly, when transformed plants (pUb1-DR-T, pUb1-DR-T, pUb1-DR-T) were challenged with $R$. solani, \% infected plants were $18.66 \%$, $21.49 \%$, and $41.64 \%$, respectively, compared to $66.33 \%$ of untransformed control plants (Fig. 5b and Table 1).

Microscopic analysis was carried out to follow the disease symptom development on inoculated leaves and roots. Tissue sections of inoculated leaves were cleaned with $6 \% \mathrm{KOH}$, stained with calcofluor white, and examined under epifluorescence microscope. In the control leaves infected with $M$. oryzae spores, extensive fungal coloniza- 
Fig. 4 Immunolocalization of Dm-AMP1 and Rs-AFP2 in roots and leaves of transgenic rice. Bar corresponds to $10 \mu \mathrm{m}$. (i) Transverse sections were prepared from root tissues of RD-T-22 and control untransformed Pusa basmati 1. a Transverse sections of transgenic plants treated with anti-Dm-AMP1 and FITCconjugated goat anti-rabbit IgG. $b$ TS of transgenic plants treated with anti-Rs-AFP2 and FITCconjugated goat anti-rabbit IgG. $c$ and $d$ control untransformed leaf tissue. (ii) Transverse sections were prepared from leaf tissues of RD-T-22 and control untransformed Pusa basmati 1; $a$ TS of transgenic plants treated with anti-Dm-AMP1 and FITCconjugated goat anti-rabbit IgG; $b$ TS of transgenic plants treated with anti-Rs-AFP2 and FITCconjugated goat anti-rabbit IgG. $c$ and $d$ control untransformed leaf tissue. (i)
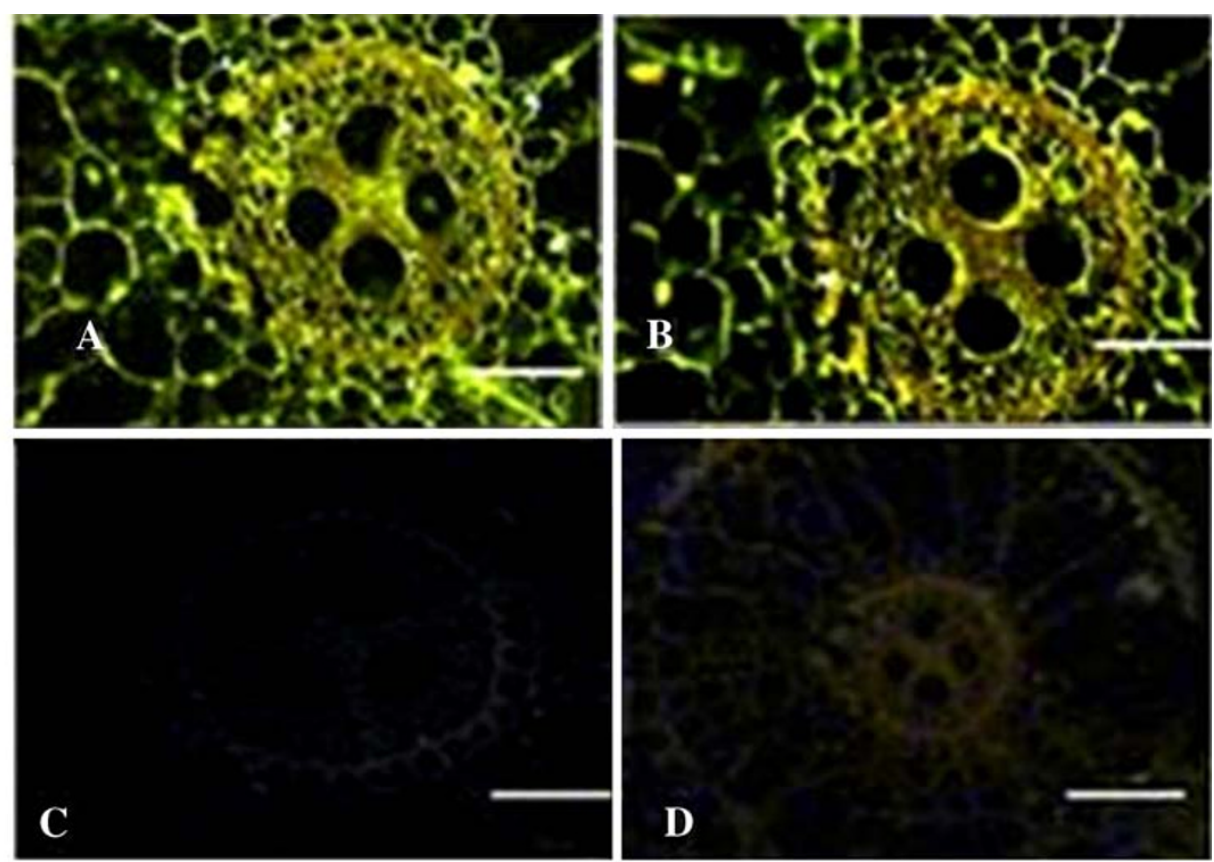

(ii)
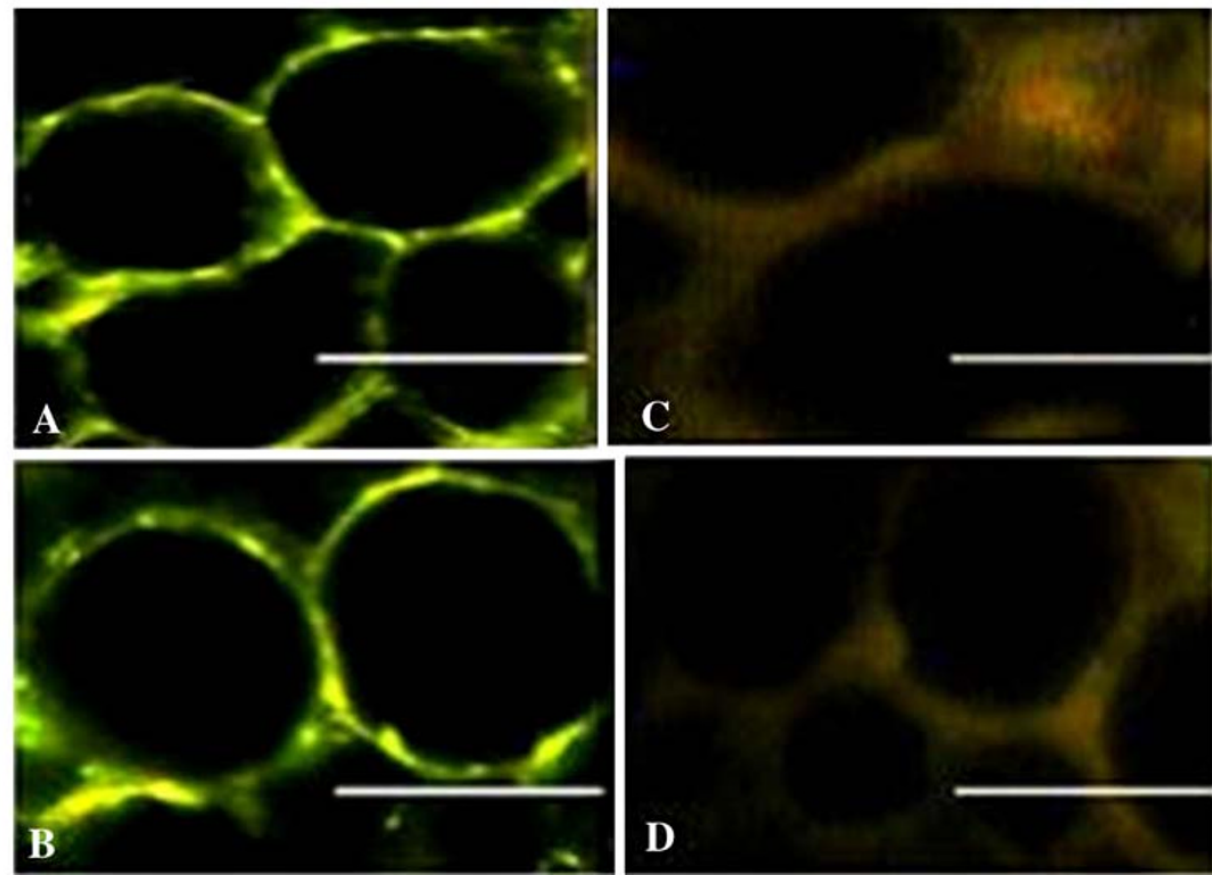

tion was observed. In contrast, reduced fungal colonization and growth inhibition were observed in the leaves of the pUbI-DR-T line under same experimental conditions (Fig. 6 (i and ii)).

Effects of total soluble protein from transgenic lines on germinating conidia of $M$. oryzae were observed by microscopy using calcofluor white. The fungal biomass treated with total soluble protein from untransformed lines showed uniform distribution of chitin and glucan and the septa were also distinctly visible. However, fungal biomass treated with total soluble protein from transgenic lines showed densely stained bulbous structures at the tip of mycelia, (Fig. 6 (i and ii)). Prolonged exposure led to increased bulging of the balloon-like structure that apparently ruptured, resulting in the release of cellular content into the surrounding medium. Rs-AFP2 as well as polyprotein showed hyper-branching and morphological abnormalities in fungal mycelium (Fig. 6 (iii)). 


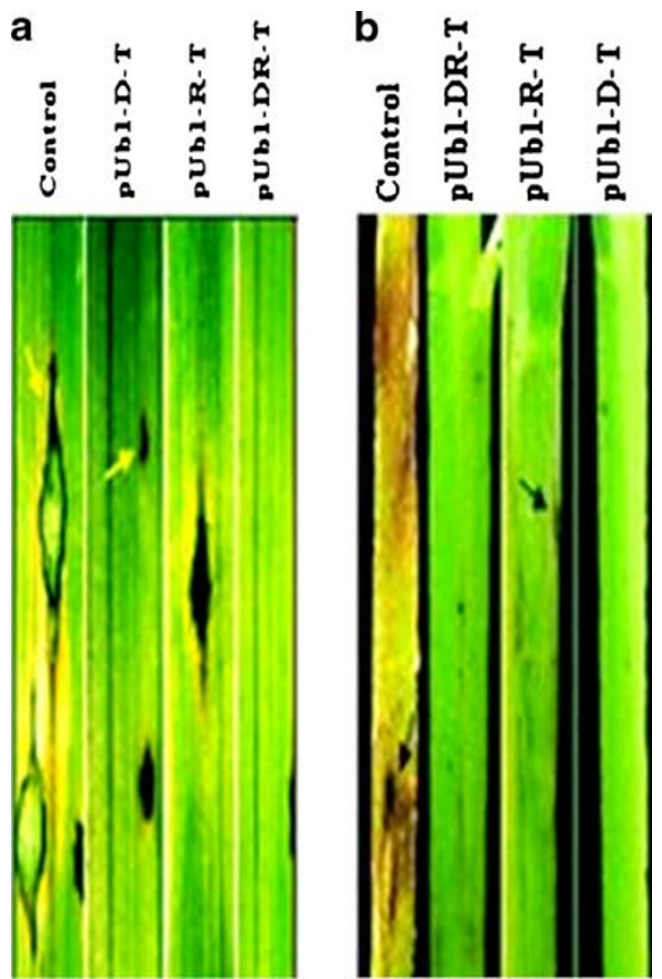

Fig. 5 a In planta bioassay of $M$. oryzae $\left(10^{5}\right.$ spores $\left./ \mathrm{ml}\right)$ on transgenic lines. Whole plant infection assay of untransformed control and pUb1-D-T, pUb1-R-T, and pUb1-DR-T transgenic rice lines. Infected leaf area was measured as \% DLA (diseased leaf area) at 10 dpi. pUb1-DR-T means DR-T-11, DR-T-6, DR-T-4, and DR-T-22 lines; pUb1-D-T means Dm-T-14, Dm-T-16, Dm-T-21, and Dm-T-32; pUb1-R-T means Rs-T-31, Rs-T-26, Rs-T-24, and Rs-T-42. b In planta bioassay of transgenic lines with $R$. solani. The number of plants showing disease symptoms was used as disease scoring system. Whole plant infection assay of untransformed control and pUb1-D-T, pUb1-R-T, and pUb1-DR-T transgenic rice lines 20 seeds, each from untransformed plants and the transgenic lines were used for sheath blight assay. pUb1-DR-T means DR-T-11, DR-T-6, DR-T-4, and DRT-22 lines; pUb1-D-T means Dm-T-14, Dm-T-16, Dm-T-21, and DmT-32; pUb1-R-T means Rs-T-31, Rs-T-26, Rs-T-24, and Rs-T-42.

\section{Discussion}

Co-expression of transgenes encoding antimicrobial proteins (AMPs) with different biochemical targets is an attractive approach to attain resistance against a broad range of pathogens. Prerequisite for the co-expression is stoichiometric and coordinated expression of two or more proteins from a single transgenic locus. Achieving coexpression of transgenes using conventional techniques results into increased number of independently assorting loci, inadequate expression levels, and problems due to gene silencing. In this study, we constructed a chimeric polyprotein containing Dm-AMP1 with native signal peptide, 16-amino-acid-linker peptide of the Ib-AMP precursor and Rs-AFP2 proteins driven by maize ubiquitin promoter and expressed it in rice. Because of its small size, the Ib-AMP precursor protein is an ideal system for coordinated expression of multiple genes. Dm-AMP1 and Rs-AFP2 show in vitro antifungal activity against several agronomically important fungal pathogens at micromolar concentration and have different biochemical targets that are important for durable and broad-spectrum resistance (Terras et al. 1995; Thevissen et al. 2004). Thus, DmAMP1 and Rs-AFP2 were selected as the candidate genes in the present study.

Expression of AMPs (with high content of disulfide bonding) in the cytosol is not an option because it will not lead to disulfide bond formation. Therefore, a secretory signal peptide (28 amino acids) of Dm-AMP1 was used to target the protein into the apoplastic region. The extracellular localization of AMPs in transgenic plants was confirmed by immunohistochemical methods using sections prepared from leaves and roots of transgenic plants. Our analysis suggested that the polyprotein signal sequence from Dm-AMP1 was recognized by the signal recognition machinery in rice and targeted both the constituent AMPs (Dm-AMP1 and Rs-AFP2) to the apoplast. This indicates that the processing of the polyprotein precursor encoded by construct pUb1-DR-T occurs in at least two steps: first removal of signal peptide as it occurs during the maturation of native Dm-AMP1, followed by cleavage at the linker peptide sequence. D'Hondt et al. (1993) isolated an aspartic endoproteinase from the seed of Brassica napus that cleaves between two consecutive acidic amino acids. The protease (or group of proteases) responsible for the cleavage of Ib-AMP1 proprotein is not known, it is possible that the carboxy terminus of the linker peptide is cleaved by an endoproteinase between two acidic amino acids, Glu and Asp. A comparison of the amino acid sequence of the five propeptides of the natural polyprotein precursor isolated from I. balsamina (Tailor et al. 1997; Francois et al. 2002) shows that linker peptides have the doublet Glu/Asp at their carboxy-terminal end. It is possible that the cleavage of this linker peptide in I. balsamina occurs between the acidic doublet Glu/Asp. The linker peptide used in constructs pUb1-DR-T contains this acidic doublet internally, which might form a recognition sequence for a specific endoproteinase. The precise point of cleavage of the linker peptide when expressed in rice plant cells remains to be determined. Since the initial interaction between the pathogen and the host occurs in the extracellular space, apoplast localization of the cystine rich AMPs was preferred.

Northern blot analysis showed a clear difference in size of the mRNA in plants transformed with the polyprotein construct as compared to plants transformed with the single-protein construct. This size difference is due to the polyprotein construct being transcribed into mRNA coding 
(i)

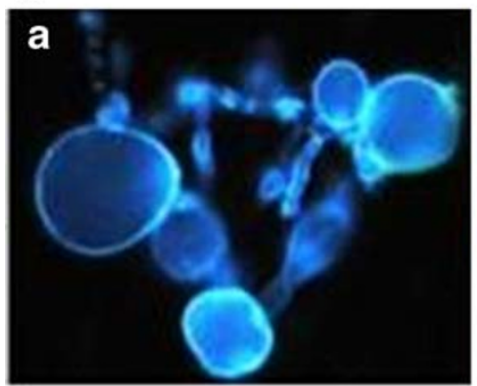

(iii)
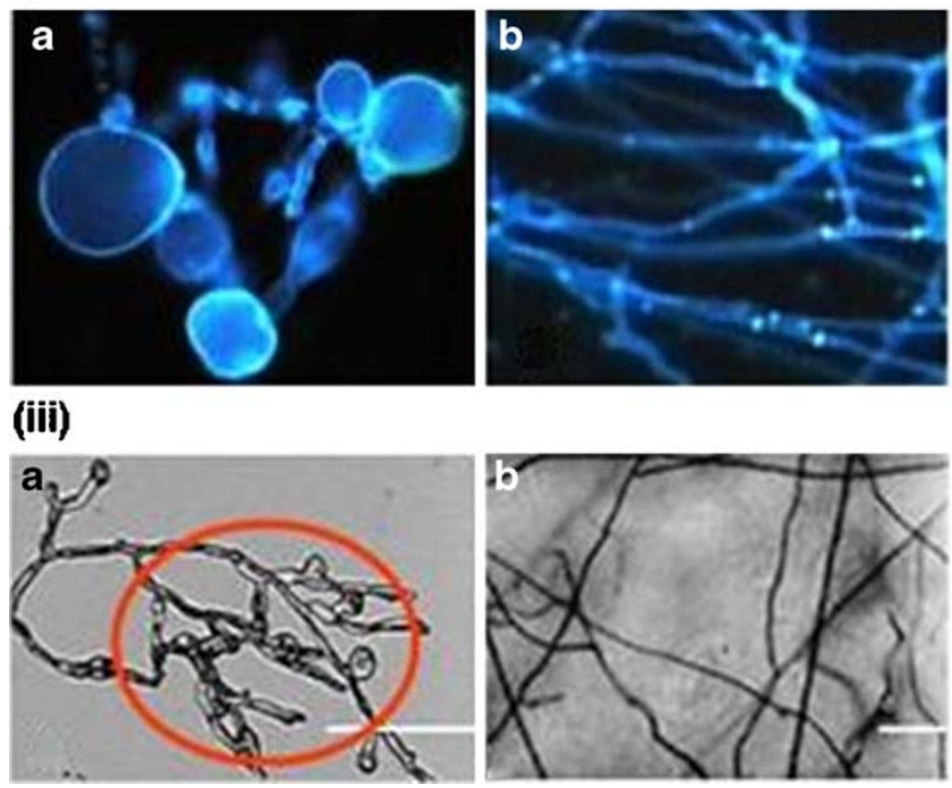

(ii)
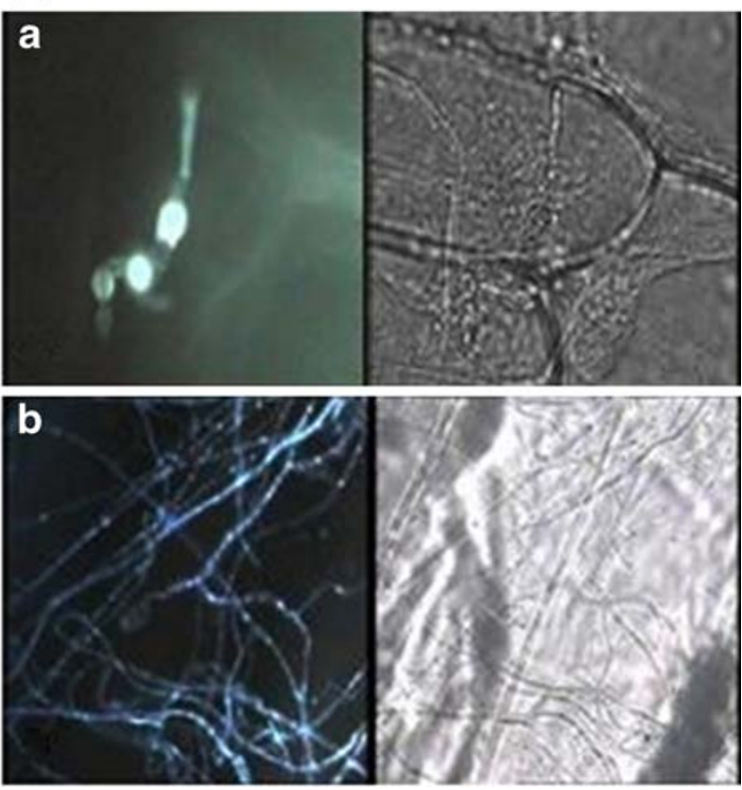

Fig. 6 (i) in vitro bioassay with total soluble protein $(20 \mu \mathrm{g})$ from DR-T-22 and untransformed Pusa basmati 1 plants; approximately, $10^{3}$ spores of $M$. oryzae $(100 \mu \mathrm{l})$ suspension were germinated on a glass slide with $20 \mu \mathrm{g}$ of total protein from leaf tissue of transgenic plants. Growth inhibition was assayed after $24 \mathrm{~h}$, in terms of percent germination or altered morphology of hyphae as compared to the control samples. The treated and untreated samples were fixed and stained with $0.3 \mathrm{~g} / \mathrm{l}$ calcofluor white examined under epifluorescence microscope. a M. oryzae treated with total soluble protein from DR-T22 plant led to bulb formation at fungal hyphal tip. $b$ M. oryzae treated with total soluble protein from untransformed plants showed uniform distribution of chitin and glucan and the septa were distinctly visible. (ii) Disease response of transformed and untransformed rice leaves challenged with $M$. oryzae spores. Twenty-one-day-old transgenic lines and untransformed control plants were inoculated with $M$. oryzae spores. Transverse sections of DR-T-22 transgenic lines and untransformed control were prepared. The samples were stained with calcofluor white. $a$ Transverse sections of transgenic leaves with its corresponding bright field image. $b$ Untransformed control with its corresponding bright field image. (iii) M. oryzae mycelia when treated with total protein from leaf tissue of transgenic plants showed abnormal morphology. Bar corresponds to $10 \mu \mathrm{m}$. a M. oryzae treated with total soluble protein from DR-T-22 plants showed hyperbranching; $b M$. oryzae treated with total soluble protein from untransformed plants showed profuse growth. for the leader peptide, Dm-AMP1, the linker peptide and Rs-AFP2, whereas the single-protein construct is transcribed as mRNA only, coding for the leader peptide and Dm-AMP1 or Rs-AFP2.

Expression level of Dm-AMP1 and Rs-AFP2 in leaf protein extracts of transgenic plants were analyzed by indirect ELISA. The levels of Dm-AMP1 in transgenic lines were in the range of $0.74 \%$ to $0.92 \%$ in polyprotein construct whereas it was $0.43 \%$ to $0.57 \%$ in singleprotein construct (Dm-AMP1) plants. Similarly, the levels for Rs-AFP2 in pUb1-DR-T plants and pUb1-R$\mathrm{T}$ plants were in the range of $0.72 \%$ to $0.87 \%$ and $0.45 \%$ to $0.53 \%$, respectively. Levels of Dm-AMP1 and RsAFP2 in plants transformed with the polyprotein construct pUb1-DR-T were not significantly different. Western blot analysis using anti-Dm-AMP1 and anti-Rs-AFP2 antibodies revealed consistent cleavage efficiency of IbAMP-linker, among individuals in different pUb-DR-T plants. Since, a single 'cleavage' within a polyprotein will result in discrete proteins in essentially equimolar quantities, coordinated expression of the two genes was observed in plants transformed with polyprotein construct, pUb1-DR-T.

A striking observation from our experiments was 2fold higher expression of Dm-AMP1 and Rs-AFP2 in pUb1-DR-T transformed lines as compared to plants transformed with single-gene construct. Although, only four transgenic lines were compared for each construct, which might not be sufficient to resolve position effects from true expression effects, but in all the polyprotein lines tested higher levels of protein expression was observed as compared to plants transformed with single-gene constructs. The fusion of a small peptide to another protein as a way to enhance its expression level has been suggested in the literature. Okamoto et al. (1998) describe enhanced expression of the gene coding for the antimicrobial peptide sarcotoxin IA by fusing translationally the coding sequence of this gene to that of Escherichia coli GUS. Western blot analysis of transgenic tobacco plants demonstrated that the amounts of sarcotoxin IA present in the form of sarcotoxin 
IA-GUS fusion proteins were considerably higher than in tobacco plants transformed with the single sarcotoxin IA peptide construct. The levels of mRNA accumulation did not differ significantly between plants transformed with the single protein and the fusion protein constructs, and therefore it was suggested that sarcotoxin IA peptide was protected from degradation when fused to GUS. We also observed that the amount of Dm-AMP1- and Rs-AFP2specific mRNA in plants transformed with the double protein construct (pUb1-DR-T) does not differ significantly from the amount of $D m-A M P 1$ and $R s-A F P 2$-specific mRNA in plants transformed with the single-protein construct (pUb1-D-T and pUb1-R-T). Protection from degradation cannot explain the observed differences at the protein level because the eventually released mature proteins are almost identical. It is possible that, because of the increased length of the mRNA in plants transformed with the double protein construct, the mRNA becomes more efficiently translated than in the case of the smaller mRNA in plants transformed with the single-protein construct, indicating that the polyprotein expression strategy might boost expression levels of small proteins.

Plant defensins fold into a small and compact $\beta$-barrel, which is stabilized by four internal disulfide bridges. Correct processing and expression of the peptide at high levels in transgenic plants is important for in planta antimicrobial activity. Our study showed that the constitutive expression of the AMPs (Dm-AMP1 and Rs-AFP2) was sufficient to inhibit the growth of $M$. oryzae and $R$. solani. pUb1-DR-T plants showed significantly improved disease resistance for $M$. oryzae and $R$. solani by $90 \%$ and $79 \%$, respectively, as compared to untransformed plants. Furthermore, reduction in infection with $M$. oryzae or $R$. solani in pUb1-DR-T transformed plants was observed as compared to pUb1-D-T or pUb1-R-T plants. Relatively higher degree of resistance observed in pUb1-DR-T plants than the plants transformed with single-protein constructs might be by boosting the amount of antimicrobial proteins produced rather than through any cooperative effect of two different proteins. Furthermore, induction of pathogenesisrelated (PR1) gene expression was not observed in either of the transgenic lines (pUb1-DR-T, pUb1-D-T, and pUb1-R-T), suggesting that the transgene product itself is directly active against the pathogens.

Constitutively expressed polyprotein genes in rice did not appear to inhibit the germination of spores, but inhibited $M$. oryzae hyphal proliferation at the infection site. As observed, small lesions did not spread to form the large lesions unlike in the control plants. We also observed a consistent difference in the severity of tissue damage between control and pUb1-DR-T transgenic plants, when monitored for the development of disease symptoms through microscopic analysis. Strong evidence of enhanced resistance due to direct effect of the overexpressed AMPs (Dm-AMP1 and Rs-AFP2) comes from growth anomalies of the fungal hyphae on transgenic lines. We found formation of bulbous structure at the hyphal tip, hyperbranching of the hyphae and an apparent collapse of the plasma membrane. Similar effects on fungal growth have been reported for fungicides (Robson et al. 1989; Wiebe et al. 1990), plant defensins (Terras et al. 1995), transgenic plants overexpressing Ace-AMP1 (Patkar and Chattoo 2006), and Mj-AMP1 (Prasad et al. 2008). In vitro and in vivo antifungal activities observed in pUb1-DR-T transgenic plants indicated that the Dm-AMP1 and Rs-AFP2 were correctly folded and processed in rice cells, resulting in enhanced resistance to $M$. oryzae and $R$. solani. Thus, the present investigation showed that co-expression of chimeric polyprotein containing Dm-AMP1 with native signal peptide; 16-amino acid-linker peptide of the Ib-AMP precursor and Rs-AFP2 proteins were efficiently cleaved into discrete biologically active proteins Dm-AMP1 and Rs-AFP2, and confers disease resistance against $M$. oryzae and R. solani.

\section{Material and methods}

\section{Plasmid construction and rice transformation}

Gene for chimeric polyprotein (Dm-AMP1 with native signal peptide, 16-amino-acid-linker peptide of the Ib-AMP precursor and Rs-AFP2 proteins) was amplified from the plasmid pFAJ3105. The amplified product was further cloned at BamHI site of pAHC17 under maize ubiquitin (Ub1) promoter (Christensen and Quail 1996) to obtain pUb1-DR. pUb1-DR was digested and cloned at HindIII site in pCAMBIA-1305.2 to get pUb1-DR-T. Similarly, for comparative purposes, individual gene constructs; $\mathrm{pUb1-D-}$ $\mathrm{T}$ (Dm-AMP1) and pUb1-R-T (Rs-AFP2) were made. Agrobacterium tumefaciens (strain LBA4404, pSB1), separately transformed with pUb1-DR-T, pUb1-D-T, and pUb1-R-T, was used to transform rice (Pusa basmati 1) calli (Hiei et al. 1994). Total DNA from various independent transgenic lines was extracted (Dellaporta et al. 1983) and screened by PCR.

Southern blot analysis

The genomic DNA $(10 \mu \mathrm{g})$ from untransformed and transgenic plants was digested with restriction enzymes $K p n \mathrm{I} / \mathrm{SacI}$; electrophoresed on $0.8 \%$ agarose gel and blotted onto nylon membrane (Hybond $\mathrm{N}^{+}$) (Amersham, Buckinghamshire, England). DNA was fixed on the membranes by UV cross-linking $\left(12 \times 10^{4} \mu \mathrm{J} / \mathrm{cm}^{2}\right)$ using UV cross-linker (Spectrolinker, Spectronics Corporation, USA). Hybridisa- 
tion probe (521-bp chimeric gene) was labeled nonradioactively using Gene Images AlkPhos Direct Labeling and Detection System as per manufacturer's instructions (Amersham Biosciences, Hongkong).

\section{Northern blot analysis}

Total RNA was isolated from the leaves of transgenic and control plants as described by Logeman et al. (1987). RNA samples were subjected to formaldehyde containing agarose gel electrophoresis and transferred to nylon membrane (Hybond-N, Amersham, Buckinghamshire, England). Hybridization was performed as described by the supplier (Amersham, Buckinghamshire, England). The fold difference in the levels of various transcripts detected was measured using ImageQuant Version 2.0 for Windows (ImageQuant, Molecular Dynamics, Amersham Biosciences, HongKong, China).

\section{Raising antibodies against Rs-AFP2}

Individual GST-fusions of Dm-AMP1 and Rs-AFP2 genes were constructed in $\mathrm{pEG}(\mathrm{KT})$ vector (Mitchell et al. 1993) and expressed in Saccharomyces cerevisiae strain, s288C. Induction and purification of the fusion protein was carried out as described by the supplier (Amersham, Buckinghamshire, England). The purified fusion protein fraction was used to raise polyclonal antibodies in rabbit. The antibody titer was estimated by indirect enzyme-linked immunosorbent assay.

\section{Western blot analysis}

Total soluble proteins were extracted in $0.1 \mathrm{M}$ phosphatebuffered saline (PBS) from transgenic plants transformed with pUb1-DR-T, pUb1-D-T, and pUb1-R-T construct. Total soluble protein was estimated by Bradford method (Bradford 1976). Samples were heated for $10 \mathrm{~min}$ at $85 \mathrm{C}$ and heatlabile denatured proteins were removed by centrifugation at $12,000 \times \mathrm{g}$. Around $20 \mu \mathrm{g}$ of total soluble proteins from each sample were separated on $15 \%$ SDS-PAGE. Purified DmAMP1 and Rs-AFP2 (50 ng) was used as a positive control, and pre-stained marker, CPM1 (Cat no: 105305; Bangalore genie Pvt Ltd, Banglore, India) for molecular weight determination in respective blots. Immunoblots were developed separately with antibody raised against Dm-AMP1 and Rs-AFP2, using the alkaline phosphatase detection method (Bangalore Genei, Bangalore, India).

\section{ELISA assay}

Total soluble protein $(20 \mu \mathrm{g})$ was extracted from leaf tissues of pUb1-DR-T, pUb1-D-T, and pUb1-R-T transgenic lines and was used as the antigen for indirect ELISA using antibodies against Dm-AMP1 and/or Rs-AFP2. Known concentrations (2-100 ng) of purified Dm-AMP1 and RsAFP2 were used as standards to plot absorbance (405 nm) versus concentration, to estimate the levels of Dm-AMP1 and Rs-AFP2 in transgenic rice lines. pUb1-DR-T means DR-T-11, DR-T-6, DR-T-4, and DR-T-22 lines; pUb1-D-T means Dm-T-14, Dm-T-16, Dm-T-21, and Dm-T-32; pUb1R-T means Rs-T-31, Rs-T-26, Rs-T-24, and Rs-T-42. Four lines from each construct and four plants of each line were taken into consideration for further characterization of disease resistance. Average values were used for comparing different transgenics unless mentioned.

Immunohistochemical localization

Young leaves and roots from transformed (DR-T-22) and control plants were fixed and embedded in paraffin. Around $8-\mu \mathrm{m}$ thick sections were prepared and processed for immunological detection of Dm-AMP1 and Rs-AFP2 proteins in pUb1-DR-T plants. Anti-Dm-AMP1 or AntiRs-AFP2 primary antibody (1:100 dilution) and FITCconjugated anti-rabbit IgG secondary antibody (Sigma Chemical Co, St Louis, MO, USA) diluted to 1:20, were used for immunological staining. The slides were observed under an epifluorescence microscope (Nikon, Eclipse 80i, Kanagawa, Japan) at $\times 200$ and $\times 400$ magnification. Control slides were treated with non-immune rabbit serum and FITC-conjugated goat anti-rabbit IgG.

\section{Rice blast assay}

Twenty-one-day-old transgenic lines (four plants of each line) and untransformed control plants were inoculated with $1 \times 10^{5}$ spores per milliliter of M. oryzae and incubated in a growth chamber maintained at $28 \mathrm{C}$ and $90 \%$ relative humidity. Blast disease development was monitored over a period of 10-days post-inoculation (dpi) and the lesions were scored using standard procedures (Bonman and Mackill 1988).

\section{Sheath blight assay}

Mycelia were harvested by vortexing in fresh, sterile PDB medium, mixed with sterile soil. Twenty-five seeds, each from untransformed plants and four transgenic lines were de-husked, surface sterilized, and placed on solid Murashige and Skoog medium containing 3\% sucrose (Jach et al. 1995). Seedlings were incubated for 3-4 weeks and then transferred to uninoculated soil for a week. Four- to fiveweek-old seedlings (20, each from untransformed and four transgenic lines) were then transferred to inoculated soil and infection was monitored. 
In vitro antifungal activity assay and microscopic studies

M. oryzae (isolate B157; IC9) was treated with either $20 \mu \mathrm{g}$ of total protein from leaf tissue of transgenic plants or $5 \mu \mathrm{g}$ of purified Dm-AMP1/Rs-AFP2, for all microscopic studies unless otherwise stated. Approximately, $10^{3}$ spores of $M$. oryzae were allowed to germinate on a glass slide in the presence or absence of Dm-AMP1 and Rs-AFP2. Growth inhibition was assayed after $24 \mathrm{~h}$, in terms of percent germination or altered morphology of hyphae in comparison to the control samples. The treated and untreated samples were air dried, fixed (10\% formaldehyde, 5\% acetic acid, and $85 \%$ ethanol for $30 \mathrm{~min}$ at room temperature) and washed thoroughly with water. The fixed samples were stained with $0.3 \mathrm{~g} / 1$ calcofluor white (SigmaAldrich, USA) for $30 \mathrm{~min}$ at room temperature. Samples were washed with $0.1 \mathrm{M}$ PBS ( $\mathrm{pH}$ 7.0) before resuspending in the mounting medium. Glycerol was used as a mounting agent for fluorescence microscopy. All samples were examined at $\times 400$ magnification under epifluorescence microscope (Eclipse 80i, 40X, Nikon, Japan), equipped with 100-W high-pressure mercury lamp (Osram HBO $103 \mathrm{~W} / 2 \mathrm{~N}$ ), and filter set for calcofluor white (UV-2A, Nikon, Japan).

\section{Statistical analysis}

Levels of Dm-AMP1 and/or Rs-AFP2 in transgenic lines, Blast and sheath blight infection assay results (\% DLA and \% infection) were compared by one-way analysis of variance (ANOVA) using cropstat program developed by IRRI. Means were compared using LSD5\%. Design of experiment was completely randomized block design (CRD).

Acknowledgments We thank Dr. B. Cammue, University of Leuven, Belgium, for providing the plasmid pFAJ3105. We also extend our thanks to Dr. Suman K. Jha, Navsari Agricultural University, Navsari, India, for helping us in statistical analysis. This work was supported by the Department of Biotechnology, Ministry of Science and Technology, Government of India.

\section{References}

Agrawal GK, Rakwal R, Jwa NS, Agrawal VP. Signaling molecules and blast pathogen attack activates rice OsPR1a and OsPR1b genes: a model illustrating components participating during defense/stress response. Plant Physiol Biochem. 2001;39:1095-1103.

Bonman JM, Mackill DJ. Durable resistance to rice blast disease. Oryza. 1988;25:103-10.

Bradford MM. A rapid and sensitive method for the quantitation of microgram quantities of protein utilizing the principle of proteindye binding. Anal Biochem. 1976;72:248-54.

Broekaert WF, Terras FR, Cammue BP, Osborn RW. Plant defensins: novel antimicrobial peptides as components of the host defense system. Plant Physiol. 1995;108:1353-8.
Broglie K, Chet I, Holliday M, Cressman R, Biddle P, Knowlton S, et al. Transgenic plants with enhanced resistance to the fungal pathogen Rhizoctonia solani. Science. 1991;254:1194-7.

Cao H, Li X, Dong X. Generation of broad-spectrum disease resistance by overexpression of an essential regulatory gene in systemic acquired resistance. Proc Natl Acad Sci U S A. 1998;95:6532-6.

Chen L, Marmey P, Taylor NJ, Brizard JP, Espinoza C, Cruz P, et al. Expression and inheritance of multiple transgenes in rice plants. Nat Biotechnol. 1998;16:1060-4.

Christensen AH, Quail P. Ubiquitin promoter-based vectors for highlevel expression of selectable and/or screenable marker genes in monocotyledonous plants. Transgenic Res. 1996;5:213-8.

Christou P. Rice transformation: bombardment. Plant Mol Biol. 1997;35:197-203.

Datta S, Muthukrisnan S, Datta SK. Expression and function of PR proteins in transgenic plants. In: Datta SK, Muthudrishan S, editors. Pathogenesis-related proteins in plants. New York: CRC; 1999. p. 261-77.

Datta K, Baisakh N, Thet KM, Tu J, Datta SK. Pyramiding transgenes for multiple resistance in rice against bacterial blight, yellow stem borer and sheath blight. Theor Appl Genet. 2002;106:1-8.

Dellaporta SL, Wood J, Hicks JB. A plant DNA minipreparation: version 2. Plant Mol Biol Rep. 1983;1:19-22.

D'Hondt K, Bosch D, Van Damme J, Goethals M, Vandekerckhove J, Krebbers E. An aspartic endoproteinase present in seeds cleaves Arabidopsis 2S albumins in vitro. J Biol Chem. 1993;268:2088491.

Francois IE, De Bolle MF, Dwyer G, Goderis IJ, Woutors PF, Verhaert $\mathrm{PD}$, et al. Transgenic expression in Arabidopsis of a polyprotein construct leading to production of two different antimicrobial proteins. Plant Physiol. 2002;128:1346-58.

Hiei Y, Ohta S, Komari T, Kumashiro T. Efficient transformation of rice (Oryza sativa L.) mediated by Agrobacterium and sequence analysis of the boundaries of the T-DNA. Plant J. 1994;6(2): 271-82.

Huang N, Angeles ER, Domingo J, Magpantay G, Singh S, Zhang G, et al. Pyramiding of bacterial blight resistance genes in rice: marker assisted selection using RFLP and PCR. Theor Appl Genet. 1997;95:313-20.

Jach G, Gornhardt B, Mundy J, Logemann J, Pinsdorf E, Leah R, et al. Enhanced quantitative resistance against fungal disease by combinatorial expression of different barley antifungal proteins in transgenic tobacco. Plant J. 1995;8(1):97-109.

Jha S, Tank HG, Prasad BD, Chattoo BB. Expression of Dm-AMP1 in rice confers resistance to Magnaporthe oryzae and Rhizoctonia solani. Transgenic Res. 2009;. doi:10.1007/s11248-008-9196-1.

Kim JK, Jang IC, Wu R, Zuo WN, Boston RS, Lee YH, et al. Coexpression of a modified maize ribosome-inactivating protein and a rice basic chitinase gene in transgenic rice plants confers enhanced resistance to sheath blight. Transgenic Res. 2003;12: 475-84.

Logeman J, Schell J, Willmitzer L. Improved method for the isolation of RNA from plant tissues. Ann Biochem. 1987;163:16-20.

Lorito M, Woo SL, Garc1'a-Fernandez I, Colucci G, Harman GE, Pintor-Toro JA, et al. Genes from mycoparasitic fungi as a source for improving plant resistance to fungal pathogens. Proc Natl Acad Sci U S A. 1998;95:7860-5.

Maqbool SB, Christou P. Multiple traits of agronomic importance in transgenic indica rice plants: analysis of transgene integration patterns, expression levels and stability. Mol Breed. 1999;5: 471-80.

Maruthasalam S, Kalpana K, Kumar KK, Loganathan M, Poovannan $\mathrm{K}$, Raja JAJ, et al. Pyramiding transgenic resistance in elite indica rice cultivars against the sheath blight and bacterial blight. Plant Cell Rep. 2007;26(6):791. 
Matzke AJ, Matzke MA. Position effects and epigenetic silencing of plant transgenes. Curr Opin Plant Biol. 1998;1:14-148.

Mew TW, Vera Cruz CM, Medalla ES. Changes in the race frequency of Xanthomonas oryzae pv. oryzae in response to the planting of rice cultivars in the Philippines. Plant Dis. 1992;76:1029-32.

Mitchell DA, Marshall TK, Deschenes RJ. Vectors for the inducible expression of glutathione S-transferase fusion proteins in yeast. Yeast. 1993;9:715-23.

Okamoto M, Mitsuhara I, Ohshima M, Natori S, Ohashi Y. Enhanced expression of an antimicrobial peptide sarcotoxin IA by GUS fusion in transgenic tobacco plants. Plant Cell Physiol. 1998;39:57-63.

Osborn RW, De Samblanx GW, Thevissen K, Goderis I, Torrekens S, Van Leuven F, et al. Isolation and characterisation of plant defensins from seeds of asteraceae, fabaceae, hippocastanaceae and saxifragaceae. FEBS Lett. 1995;368:257-62.

$\mathrm{Ou} \mathrm{SH}$. Rice diseases. Commonwealth mycological institute publication. Second ed. Surrey: Kew; 1985. p. 280-2.

Patkar RN, Chattoo BB. Transgenic indica rice expressing ns-LTP like protein shows enhanced resistance to both fungal and bacterial pathogens. Mol Breed. 2006;17:159-71.

Prasad BD, Jha S, Chattoo BB. Transgenic indica rice expressing Mirabilis jalapa antimicrobial protein (Mj-AMP2) shows enhanced resistance to the rice blast fungus Magnaporthe oryzae. Plant Sci. 2008;175:364-71.

Robson GD, Kuhn PJ, Trinci APJ. Effect of validamycin A on the inositol content and branching of Rhizoctonia cerealis and other fungi. J Gen Microbiol. 1989;135:739-50.

Ronald PC. The molecular basis of disease resistance in rice. Plant Mol Biol. 1997;35:179-86.

Song WY, Wang GL, Chen LL, Kim HS, Pi LY, Hosten T, et al. A receptor kinase-like protein encoded by the rice disease resistance gene Xa21. Science. 1995;270:1804-6.

Tailor RA, Acland DP, Attenborough S, Cammue BPA, Evans IJ, Osborn RW, et al. A novel family of small cysteine-rich antimicrobial peptides from seeds of Impatiens balsamina is derived from a single precursor protein. J Biol Chem. 1997;272:24480-7.
Takakura Y, Ito T, Saito H, Inoue T, Komari T, Kuwata S. Flowerpredominant expression of a gene encoding a novel class I chitinase in rice (Oryza sativa L.). Plant Mol Biol. 2000;42:883-97.

Terras FRG, Schoofs HME, De Bolle MFC, Van Leuven F, Rees SB, Vanderleyden $\mathrm{J}$, et al. Analysis of two novel classes of plant antifungal proteins from radish (Raphanus sativus L.) seeds. J Biol Chem. 1992;267:15301-9.

Terras FRG, Eggermont K, Kovaleva V, Raikhel NV, Osborn RW, Kester A, et al. Small cysteine-rich antifungal proteins from radish: their role in host defence. Plant Cell. 1995;7:573-88.

Thevissen K, Warnecke DC, François IEJA, Leipelt M, Heinz E, Ott $\mathrm{C}$, et al. Defensins from insects and plants interact with fungal glucosylceramides. J Biol Chem. 2004;279:3900-5.

Thomma BPHJ, Cammue BPA, Thevissen K. Plant defensins. Planta. 2002;216:193-202.

Van Loon LC, Van Strien EA. The families of pathogenesis-related proteins, their activities, and comparative analysis of PR-1 type proteins. Physiol Mol Plant Pathol. 1999;55:85-97.

Velazhahan R, Chen-Cole K, Anuratha CS, Muthukrishnan S. Induction of thaumatin-like proteins (TLPs) in Rhizoctonia solani infected rice and characterization of two new cDNA clones. Physiol Plant. 1998;102:21-8.

Wiebe MG, Robson GD, Trinci APJ. Edifenphos (Hinosan) reduces hyphal extension, hyphal growth unit length and phosphatidylcholine content of Fusarium graminearum A3/5, but has no effect on specific growth rate. J Gen Microbiol. 1990;136:97984.

Ye XD, Al-Babili S, Kloti A, Zhang J, Lucca P, Beyer P, Potrykus I. Engineering the pro-vitamin A ( $\beta$-carotene) biosynthetic pathway into (carotenoid free) rice endosperm. Science. 2000; 287:303-5.

Yun DJ, Ibeas JI, Lee H, Coca MA, Narasimhan ML, Uesono Y, et al. Osmotin, a plant antifungal protein, subverts signal transduction to enhance fungal cell susceptibility. Mol Cell. 1998;1:807-12.

Zhang S, Song WY, Chen L, Ruan D, Taylor N, Ronald PC, et al. Transgenic elite indica rice varieties, resistant to Xanthomonas oryzae pv.oryzae. Mol Breed. 1998;4:551-8. 